\title{
Bioinformatics and Functional Analyses Implicate Potential Roles for EOGT and $L$-fringe in Pancreatic Cancers
}

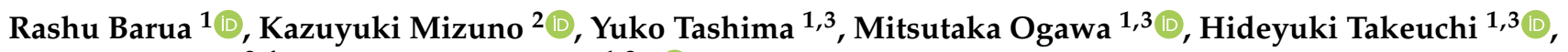 \\ Ayumu Taguchi ${ }^{2,4}$ and Tetsuya Okajima $1,3, *$ D \\ 1 Department of Molecular Biochemistry, Nagoya University Graduate School of Medicine, 65 Tsurumai, \\ Showa-ku, Nagoya 466-8550, Japan; rashubarua2013@gmail.com (R.B.); tashima@med.nagoya-u.ac.jp (Y.T.); \\ mitsutaka.ogawa@med.nagoya-u.ac.jp (M.O.); htakeuchi@med.nagoya-u.ac.jp (H.T.) \\ 2 Division of Molecular Diagnostics, Aichi Cancer Center, 1-1 Kanokoden, Chikusa-ku, Nagoya, \\ Aichi 464-8681, Japan; k.mizuno@aichi-cc.jp (K.M.); a.taguchi@aichi-cc.jp (A.T.) \\ 3 Institute for Glyco-core Research (iGCORE), Integrated Glyco-Biomedical Research Center, \\ Nagoya University, Furo-cho, Chikusa-ku, Nagoya 464-8601, Japan \\ 4 Division of Advanced Cancer Diagnostics, Nagoya University Graduate School of Medicine, \\ 65 Tsurumai, Showa-ku, Nagoya 466-8550, Japan \\ * Correspondence: tokajima@med.nagoya-u.ac.jp; Tel.: +81-52-744-2068; Fax: +81-52-744-2069
}

Citation: Barua, R.; Mizuno, K.;

Tashima, Y.; Ogawa, M.; Takeuchi, H.;

Taguchi, A.; Okajima, T.

Bioinformatics and Functional Analyses Implicate Potential Roles for EOGT and $L$-fringe in Pancreatic Cancers. Molecules 2021, 26, 882. https://doi.org/10.3390/ molecules 26040882

Academic Editor: Paola Arimondo Received: 5 January 2021

Accepted: 2 February 2021

Published: 7 February 2021

Publisher's Note: MDPI stays neutral with regard to jurisdictional claims in published maps and institutional affiliations.

Copyright: (c) 2021 by the authors. Licensee MDPI, Basel, Switzerland. This article is an open access article distributed under the terms and conditions of the Creative Commons Attribution (CC BY) license (https:/ / creativecommons.org/licenses/by/ $4.0 /)$.

\begin{abstract}
Notch signaling receptors, ligands, and their downstream target genes are dysregulated in pancreatic ductal adenocarcinoma (PDAC), suggesting a role of Notch signaling in pancreatic tumor development and progression. However, dysregulation of Notch signaling by post-translational modification of Notch receptors remains poorly understood. Here, we analyzed the Notch-modifying glycosyltransferase involved in the regulation of the ligand-dependent Notch signaling pathway. Bioinformatic analysis revealed that the expression of epidermal growth factor (EGF) domain-specific $O$-linked $\mathrm{N}$-acetylglucosamine (EOGT) and Lunatic fringe (LFNG) positively correlates with a subset of Notch signaling genes in PDAC. The lack of EOGT or LFNG expression inhibited the proliferation and migration of Panc- 1 cells, as observed by the inhibition of Notch activation. EOGT expression is significantly increased in the basal subtype, and low expression of both EOGT and LFNG predicts better overall survival in PDAC patients. These results imply potential roles for EOGT- and LFNGdependent Notch signaling in PDAC.
\end{abstract}

Keywords: EOGT; LFNG; Notch; PDAC

\section{Introduction}

Pancreatic cancer is one of the most fatal malignancies, which causes cancer mortality in most developed countries. It was the fourth leading cause of cancer death in 2017 in the United States, and approximately 57,000 adults were diagnosed with pancreatic cancer in 2020, which represented approximately $3 \%$ of the total cancer cases [1,2]. Generally, the 5 -year survival rate of pancreatic cancer is approximately $4 \%$ [3]. More than $90 \%$ of pancreatic cancer is considered pancreatic ductal adenocarcinoma (PDAC) [4]. Typically, PDAC is diagnosed at a later stage of the disease, with highly aggressive malignancies. For this reason, patients do not receive adequate surgical treatment or conventional chemotherapy [5]. Therefore, it is necessary to determine the underlying molecular mechanism of tumor growth in PDAC to develop new therapeutic techniques that can prevent tumor progression.

The Notch signaling pathway is an evolutionarily conserved, intercellular signaling pathway involved in the cell fate decision in many biological contexts, and plays a vital role in oncogenesis [6,7]. Accordingly, Notch signaling is considered a molecular target for different malignancies $[8,9]$. In mouse models, both activation and inhibition of Notch signaling promote PDAC development and progression [10-13]. There are four types of Notch receptors (Notch 1-4) and five types of canonical Notch ligands: Delta-like 1 
(DLL1), DLL3, DLL4, Jagged 1 (JAG1), and JAG2. The interaction of epidermal growth factor (EGF)-like repeats of Notch receptors with their ligands initiates the Notch signaling pathway [14]. Most of the EGF repeats are post-translationally modified by $O$-glycan, such as $\mathrm{O}$-glucose (O-Glc), $\mathrm{O}$-fucose (O-Fuc), and $\mathrm{O}$-linked $\mathrm{N}$-acetylglucosamine (O-GlcNAc) [7]. Notch signaling is a crucial factor in the progression of PDAC. It is also responsible for the EMT phenotype and cancer stem cell formation in PDAC. It has also been noted that Notch signaling components and their target genes are unregulated in invasive PDAC [15-20]. Nonetheless, dysregulated Notch signaling by the post-translational modification of Notch receptors remains poorly understood, with little research focusing on the glycosyltransferases modulating Notch activity.

EGF domain-specific O-GlcNAc-transferase (EOGT) is an endoplasmic reticulum (ER)-specific enzyme, which adds an O-GlcNAc moiety to the extracellular domain of secreted or membrane proteins, including Notch receptors and their ligands [21,22]. Evidence suggests that the mutation of EOGT causes a rare congenital disease, Adams-Oliver syndrome [23-25]. EOGT is highly expressed in endothelial cells and regulates optimal vascular integrity and development by enhancing DLL ligand-mediated Notch signaling $[24,26]$. However, there is no evidence showing that EOGT-mediated O-GlcNAcylation participates in the development and progression of cancer, including PDAC.

In addition to EOGT, Fringe genes are involved in the regulation of ligand-dependent Notch signaling pathway. Fringe encodes $\beta-1,3-$ GlcNAc-transferase modifying $O$-Fuc on Notch receptors. In mammals, Fringe activity is mediated by three different genes (LFNG, RFNG, and MFNG), of which LFNG has significantly higher catalytic activity than others [27]. Unlike EOGT, LFNG enhances Delta-ligand-mediated Notch signaling but inhibits JAG1-mediated Notch signaling [28]. Though some evidence has shown that loss of $L f n g$ in mice accelerated Kras-induced pancreatic cancer development, the roles of LFNG in human cancer cells are cell type-dependent and not fully understood [29].

To examine the contribution of EOGT and LFNG in PDAC, we conducted database analysis and functional studies in a PDAC cell line. Our study indicated critical roles for EOGT- and LFNG-dependent Notch signaling in PDAC. Furthermore, we showed-for the first time- that EOGT impacts cell proliferation and migration in cancer cells.

\section{Results and Discussion}

\subsection{Contribution of EOGT and LFNG to Notch Signaling in PDAC}

Notch target gene expression is dysregulated in PDAC $[15,16,19,20]$. To date, the expression levels of EOGT and FNG genes have been experimentally shown to affect both $O$-glycan structures and functions in mammals [26,28,30-33]. To investigate the expression of EOGT and FNG genes in PDAC, in silico analysis was performed using the GEPIA2 integrated database, which includes 179 tumor and 171 normal tissue samples. Among Notch target genes, HES1 and HEY1 expressions were higher in both basal and classical subtypes of PDAC. In contrast, EOGT expression was significantly increased in the basal subtype, which represents a more aggressive phenotype [34]. LFNG expression was not significantly altered (Figure 1A). However, these data did not exclude the possibility of multiple, rather than a single, glycosyltransferase(s) contributing to dysregulated Notch signaling in PDAC.

Previous studies demonstrated that the loss of EOGT decreased DLL ligand-induced Notch signaling and downstream Notch target genes [26]. Thus, we analyzed the correlation between EOGT and the upregulated Notch target genes (Figure 1B). Spearman correlation coefficient was used to assess monotonic correlations. Correlation analysis revealed a significant positive correlation between EOGT and HEY1 $\left(r=0.47, p=4.2 \times 10^{-11}\right)$. In contrast, the correlation analysis between $L F N G$ and NOTCH target genes revealed a positive correlation between LFNG and HES1 expression $\left(r=0.48, p=8.4 \times 10^{-12}\right)$. A negligible correlation was observed for MFNG and RFNG [28]. These data suggested that the contribution of EOGT and LFNG to Notch signaling is qualitatively different, possibly through the differential impact on multiple Notch-ligand pairs, which leads to the trans- 
activation of distinct sets of Notch signaling target genes, including HES1 and HEY1 [35]. Further in-depth bioinformatics analyses included Biclustering methods, will help elucidate the pathological relevance of the observed correlations in tumor progression [36-39].

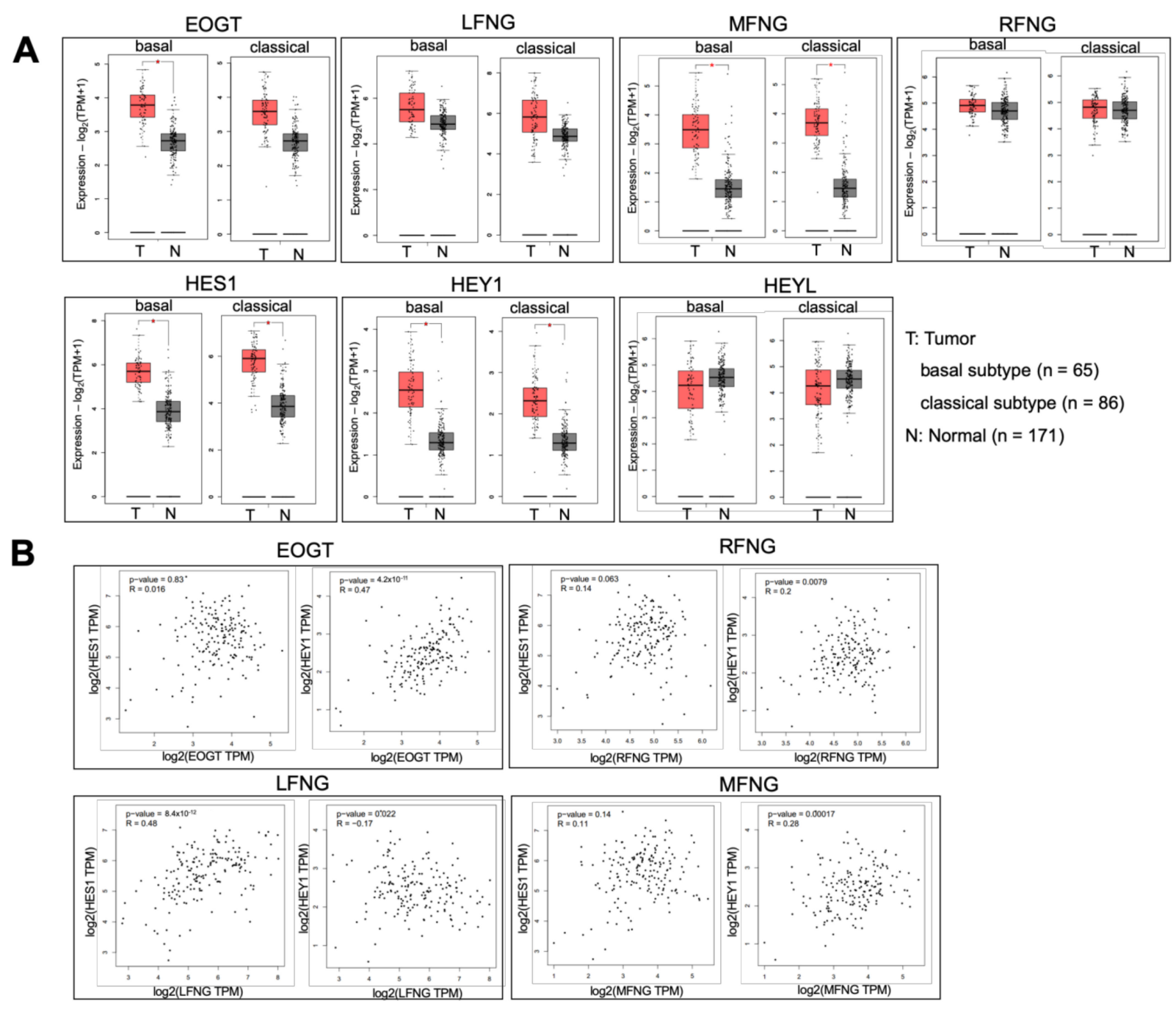

Figure 1. Expression of epidermal growth factor (EGF) domain-specific $O$-linked $N$-acetylglucosamine (EOGT) and Lunatic fringe $(L F N G)$ in pancreatic ductal adenocarcinoma (PDAC) analyzed by the GEPIA2 database. (A) Gene expression patterns between normal and PDAC tissues analyzed using the GEPIA2 database. ${ }^{*} p<0.01$. Red bars indicate PDAC tissues (basal or classical subtypes), and gray bars normal tissues (TCGA normal and GTEx data). (B) The correlation in gene expression between glycosyltransferase genes (EOGT, LFNG, RFNG, or MFNG) and Notch target genes (HES1 or HEY1) was analyzed using the GEPIA2 database.

\subsection{Expression of EOGT in PDAC Cell Lines}

We compared endogenous expression levels of EOGT in human PDAC cell lines to a normal pancreatic ductal cell line, H6C7. From immunoblotting data, we found that four PDAC cell lines (Panc-1, BxPC3, Panc03.27, and CAPAN-2) out of ten showed higher EOGT expression compared to $\mathrm{H} 6 \mathrm{C} 7$ cells (Figure 2A). We also verified the expression of EOGT by immunostaining in the four PDAC cell lines (Figure 2B). Based on the immunoblotting and immunostaining assays, we selected Panc-1, which showed prominent EOGT expression, for functional analysis. 

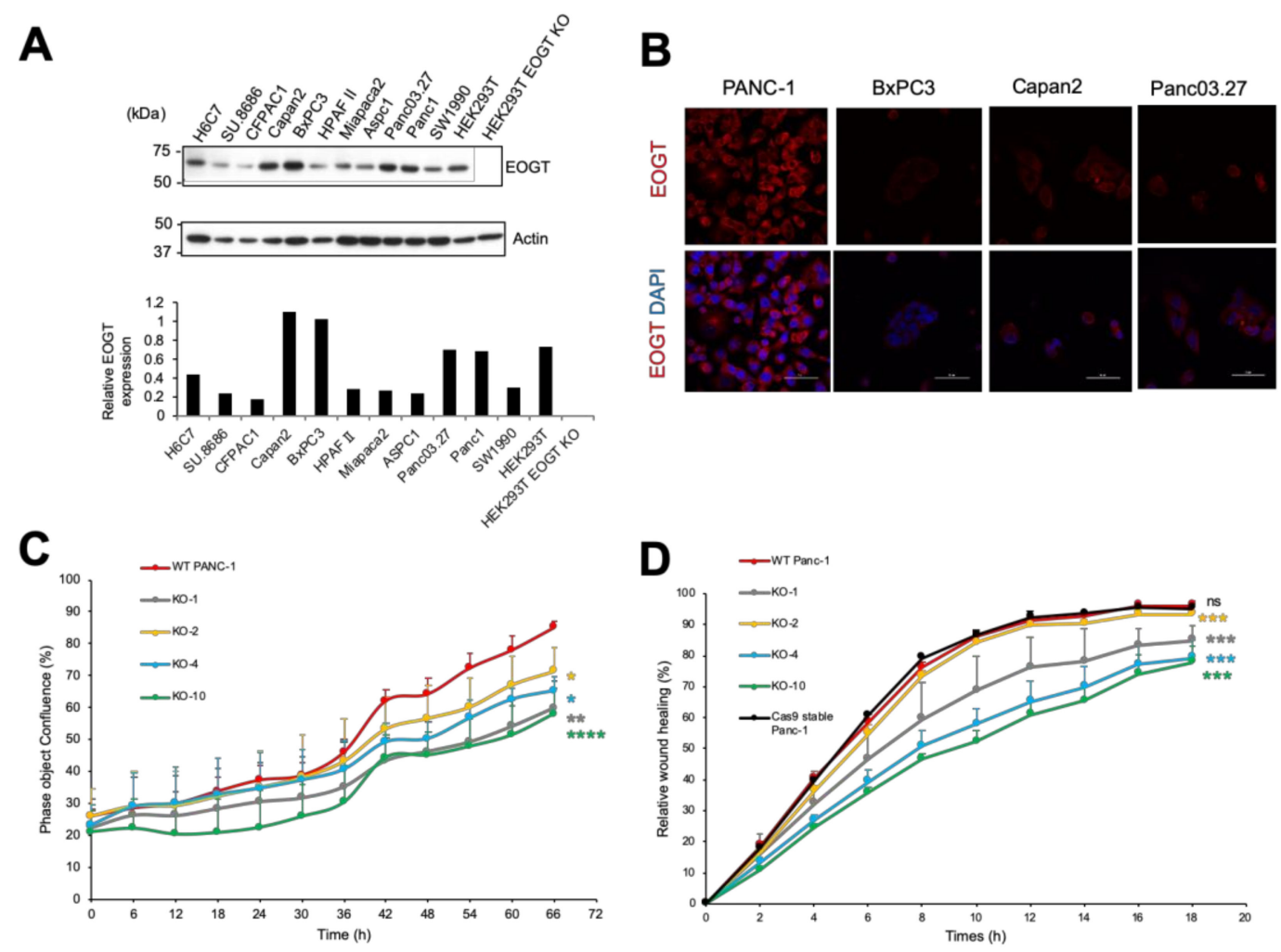

Figure 2. EOGT promotes the proliferation and migration of Panc-1 cells. (A) Cell lysates prepared from different pancreatic cancer cells were analyzed in parallel with cell lysates from HEK293T and HEK293T EOGT-KO cells. Immunoblotting was performed using anti-AER61 or beta-actin antibodies. EOGT expression level was normalized to the expression of beta-actin (below). (B) Detection of endogenous EOGT with EOGT-specific anti-AER61 (Red) antibody. Scale bar, $50 \mu \mathrm{m}$. (C) Wild-type (WT) and EOGT-KO Panc-1 cells were grown, and cell proliferation was measured by the IncuCyte ZOOM system. The data were expressed as mean \pm standard deviation (SD) of triplicate samples. $\left({ }^{* *} p=0.0018\right.$ in WT vs. KO- 1 , ${ }^{*} p=0.0215$ in WT vs. KO-2, ${ }^{*} p=0.0154$ in WT vs. KO-4, and ${ }^{* * * *} p<0.0001$ in WT vs. KO-10) (D) Cell migration assay was performed using the IncuCyte ZOOM system. At different time points, relative wound density of WT and EOGT-KO Panc-1 cells were measured for $18 \mathrm{~h}$. The measurements are from wounds made on a monolayer of cultured cells. Each data point is expressed as mean $\pm \mathrm{SD}(\mathrm{n}=3) .{ }^{* * *} p=0.0009$ in WT vs. KO-1, ${ }^{* * * *} p=0.0004$ in WT vs. KO-2, ${ }^{* * *} p=0.008$ in WT vs. KO-4, ${ }^{* * *} p=0.0007$ in WT vs. KO-10, and $p=0.7568$ in WT vs. Cas9-transfected control cells (Cas9 stable Panc-1).

\subsection{CRISPR/CAS9-Mediated Lentiviral Knockout of EOGT in a PDAC Cell Line}

To assess the role of $E O G T$ in the growth of the PDAC cell line, we performed gene editing with the CRISPR/Cas9-mediated lentivirus technique in Panc-1 cells. After lentiviral transduction and blasticidin $S$ selection, the lack of EOGT was confirmed by the anti-EOGT (AER61) antibody (Supplementary Figure S1).

\subsection{EOGT Knockout in Panc-1 Cells Impairs Cell Proliferation and Migration}

To investigate whether the decreased Notch activity inhibits Panc-1 cell proliferation, Panc-1 cells were treated with DAPT, a $\gamma$-secretase inhibitor that blocks Notch signaling. As shown in Supplementary Figure S2, the inhibitory effect of DAPT on the proliferation of Panc-1 cells was observed in a time-dependent manner. This result suggested that decreased Notch activity can substantially reduce the cell proliferation of Panc- 1 . These data agree with previous reports showing a similar effect on cell proliferation by DAPT treatment [40,41].

To examine the effect of knockout of EOGT in the Panc- 1 cells, we observed the proliferation between wild-type and EOGT-KO cells at an interval of $6 \mathrm{~h}$ over $66 \mathrm{~h}$. EOGT$\mathrm{KO}$ cells exhibited slow growth relative to wild-type parental control cells. This result showed that EOGT promotes cell proliferation in Panc-1 cells (Figure 2C). 
It has been reported that inhibition of Notch1 affects cancer cell migration and invasion in vitro in pancreatic cancer $[42,43]$. We analyzed the cell motility of the parental wildtype and EOGT-KO Panc-1 by wound-healing migration assay. Analysis of the relative wound-healing density revealed that EOGT-KO Panc-1 showed significant decreases in re-capturing the wound portion compared to wild-type control cells (Figure 2D). Compared to the control cells, EOGT-KO-1, KO-2, KO-4, KO-10 clones showed a slower migration rate, presenting a depletion of wound density $13,4,18$, and $20 \%$, respectively, within $18 \mathrm{~h}$. The differences in the migration rate among $\mathrm{KO}$ clones appear to be due to cancer cells' clonal variation. According to previous studies, the doubling time of Panc- 1 is $52-56 \mathrm{~h}$. Therefore, the effects on the wound-healing density were predominantly due to decreased cell migration of EOGT-KO Panc-1 cells, but not to decreased proliferation [44,45].

\subsection{CRISPR/Cas9-Mediated Lentiviral Knockout of LFNG in a PDAC Cell Line}

To compare the effect of EOGT and LFNG in the growth and motility of Panc-1 cells, we generated LFNG-KO cells by CRISPR/Cas9-mediated lentivirus transduction. The cutting efficacy was analyzed by T7 endonuclease assay. Then, by limiting dilution, single-cell clones were isolated. GFP-positive clones were selected for sequencing to confirm LFNG knockout in Panc-1 cells (Supplementary Figure S3).

\subsection{LFNG Knockout in Panc-1 Cells Impairs Cell Proliferation and Migration}

To evaluate the role of LFNG in cell proliferation, Panc-1 cells lacking LFNG were analyzed by the IncuCyte ZOOM system (Sartorius, Japan). The result showed that the cell proliferation of LFNG-KO cells was lower than that of parental control cells (Figure 3A). These data suggested that LFNG promotes cell proliferation of Panc-1 cells. According to previously reported data, a similar experiment was performed by shRNA-mediated knockdown of LFNG [29]. Although this group reported the tumor-suppressive behavior of LFNG in mice and pancreatic cancer cells, our findings suggested that LFNG promotes the proliferation activity of Panc-1 cells, which was also noted in the same study [29].

A

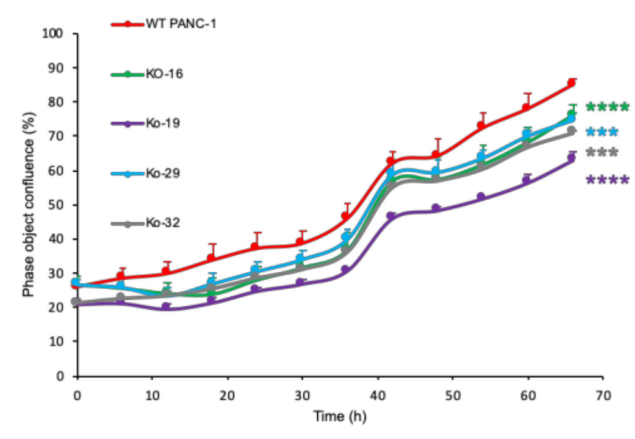

B

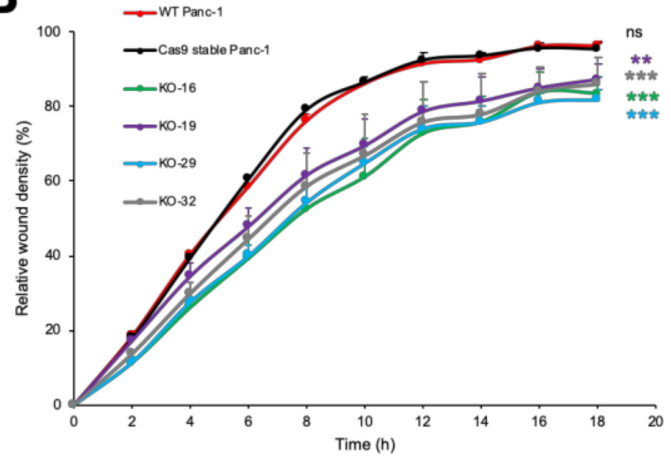

Figure 3. LFNG promotes the proliferation and migration of Panc-1 cells. (A) Wild-type (WT) and LFNG-KO Panc-1 cells were subjected to a proliferation assay with the IncuCyte ZOOM system. The data are expressed as mean \pm standard deviation (SD) of triplicate samples. ${ }^{* * * *} p<0.0001$ in WT vs. KO-16, ${ }^{* * * *} p<0.0001$ in WT vs. KO-19, ${ }^{* * *} p=0.0001$ in WT vs. KO-29, and ${ }^{* * * *} p<0.0001$ in WT vs. KO-32) (B) Cell migration of WT and LFNG-KO Panc-1 was determined by the IncuCyte ZOOM system. Each data point is expressed as mean $\pm \mathrm{SD}(\mathrm{n}=3)$. ${ }^{* * *} p=0.0006$ in WT vs. KO-16, ${ }^{* * *} p=0.0017$ in WT vs. KO-19, ${ }^{* * *} p=0.0003$ in WT vs. KO-29, ${ }^{* * *} p=0.0005$ in WT vs. KO-32, and $p=0.7568$ in WT vs. Cas9-transfected control cells (Cas9 stable Panc-1).

Then, we used the wound-healing assay to compare the motility of wild-type and LFNG-KO Panc-1 cells. The relative wound density revealed that the cell migration rate in controls was $10-15 \%$ faster than LFNG-KO Panc-1 cells (Figure 3B). These results suggest that LFNG promotes cell migration in a similar way to EOGT. 


\subsection{Low Expression of Both EOGT and LFNG Predicts Better Overall Survival in PDAC Patients}

Knowing that EOGT and LFNG modulate cell proliferation and migration, the Cancer Genome Atlas (TCGA) dataset was analyzed to evaluate the clinical outcome. For overall survival, PDAC patients were classified into high and low expression groups using the median expression of genes as the cut-off value. Kaplan-Meier survival curves revealed no association between EOGT expression and overall survival in PDAC (Figure 4A). Additionally, no association was observed between LFNG expression and overall survival (Figure 4B). In contrast, lower expression of both EOGT and LFNG is associated with a better prognosis of PDAC $(p=0.0195)$. These data suggest that the dysregulation of Notch signaling mediated by high expression of either EOGT or LFNG leads to poor prognosis of PDAC.
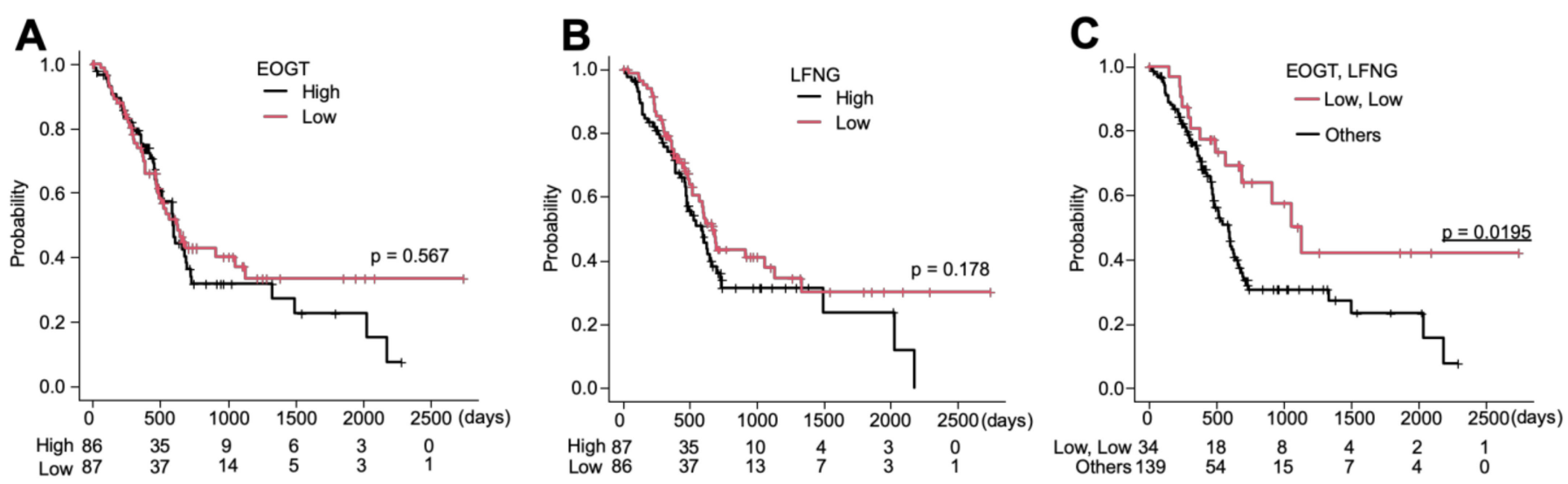

Figure 4. Kaplan-Meier curves showing the overall survival analysis in PDAC patients with high and low expression of EOGT and LFNG. (A) Association between overall survival and EOGT expression. (B) Association between overall survival and LFNG expression. (C) Lower EOGT and LFNG expression are associated with better overall survival. $p=0.0195$, by log-rank test.

In summary, we showed the roles of EOGT in cell proliferation and migration in cancer cells. In our study, loss of EOGT or LFNG resulted in a similar cellular phenotype, suggesting that both genes cooperate in the tumor properties of PDAC cells. Furthermore, the combination of lower LFNG and EOGT expression in PDAC serves as an excellent prognostic marker, implying that both Notch modifiers modulate disease progression. Given that EOGT and LFNG modulate ligand-induced Notch signaling, these results suggest that changes in the expression of these glycosyltransferases result in altered Notch activity, as indicated by the correlation between LFNG and HES1, or EOGT and HEY1 expression in the PDAC database. Therefore, dysregulated Notch signaling mediated by changes in the expression of EOGT and LFNG would affect the prognosis of PDAC. Although this study implicates potential roles for EOGT- and LFNG-dependent Notch signaling in PDAC, further studies will be necessary to clarify the roles of Notch-modifying enzymes in dysregulated Notch activity and the development and progression of PDAC at the molecular level.

\section{Materials and Methods}

\subsection{Antibodies and Reagents}

The antibodies used were the following: anti-AER61 (EPR12944) (ab190693, Abcam, Japan), anti-rabbit IgG HRP-linked antibody (\#7074, Cell Signaling, Japan), DyLight 549conjugated goat anti-rabbit IgG antibody (DI-1549, Vector Laboratories, USA). The reagents used were the following: LentiX concentrator (TaKaRa, Japan), blasticidin $\mathrm{S}$ (Funakoshi, Japan), puromycin (MERCK, Japan), doxycycline (TaKaRa, Japan), DMSO (FUJIFILM Wako, Japan), T7 endonuclease I reaction mix (Nippon Gene, Japan). Primers specific for EOGT 
and LFNG, CRISPR/Cas9 sgRNA, and G-blocks were purchased from Integrated DNA Technologies (IDT, USA).

\subsection{Cell Culture}

Panc-1, LentiX-293T, HEK293T, and HEK293T EOGT-KO cells were cultured in Dulbecco's modified Eagle's medium (DMEM) supplemented with $10 \%$ fetal bovine serum (FBS). BxPC3, Panc03.27, and Capan-2 were maintained in RPMI medium supplemented with $10 \%$ FBS.

\subsection{Vectors}

pLP1, pLP2, pVSV-G were purchased from Thermo Fisher, Japan. pLX-sgRNA (\#50662), pLRG2.1 (\#108098), and doxycycline-inducible pCW-Cas9 (\#50661) lentivirus vectors were from Addgene, USA.

\subsection{Cell growth inhibition by DAPT}

Panc- 1 cells $\left(3 \times 10^{4}\right)$ were seeded in a 24 -well plate with $500 \mu \mathrm{l}$ of DMEM/10\% FBS. After $24 \mathrm{~h}$ incubation, cells were treated with $5 \mu \mathrm{M}, 50 \mu \mathrm{M}$, and $75 \mu \mathrm{M}$ DAPT over $69 \mathrm{~h}$. Control Panc- 1 cells were treated with $0.1 \%$ DMSO in the culture medium. Cell growth inhibition was measured using IncuCyte ZOOM Live-Cell Analysis System (Sartorius) [46]. Data were analyzed using phase object confluence, which quantified the cell surface area as confluence values. The images of the cells were taken every $3 \mathrm{~h}$ with a $4 \times$ objective.

\subsection{Lentivirus Vector Construction}

For the EOGT sgRNA lentivirus vector construction, EOGT sgRNA was designed using online software CRISPRdirect [47]. The sgRNA was synthesized as a g-block segment, which includes XhoI restriction site, U6 promoter region, sgRNA target site, chimeric sgRNA scaffold, and NheI restriction site, as shown in Supplementary Table S1. The gblock was cloned into a pLX-sgRNA lentivirus vector containing blasticidin $S$-resistance gene [48]. For selecting LFNG sgRNA, we used previously validated sgRNA for $L F N G$ (Supplementary Table S1) [49]. The sgRNA sequence was inserted into the pLRG2.1 vector, which contains GFP as a selection marker [50]. A separate doxycycline-inducible FLAGCas9 expression vector ( $\mathrm{pCW}$-Cas9) was also used, which contains a puromycin resistance gene [48].

\subsection{CRISPR/Cas9-Mediated Lentiviral Knockout of EOGT and LFNG Genes}

The knockout experiments in Panc-1 cells were performed as described before with a slight modification [50,51]. Briefly, lentiviral plasmids are co-transfected with LP1, LP2, and VSVG virus-packaging plasmid in LentiX-293T cells using PEI MAX (Polysciences, USA). After $12 \mathrm{~h}$ later, culture media were replaced with a high FBS containing medium (DMEM/40\% FBS), which did not contain penicillin and streptomycin. After 24 and $48 \mathrm{~h}$ later, lentivirus supernatant was collected, centrifuged at 12,000 rpm for $5 \mathrm{~min}$, and filtered using a $0.45 \mu \mathrm{m}$ filter. After filtration, the virus was concentrated by LentiX concentrator according to the manufacturer protocol. Cas9-transfected Panc-1 cells were generated after selection with $2 \mu \mathrm{g} / \mathrm{mL}$ puromycin. Anti-FLAG antibody was used to verify Cas9 protein expression by immunoblotting. For generating EOGT-KO Panc-1 cells, the Cas 9 stable Panc-1 cells were infected with lentiviral supernatant, which contains EOGT sgRNA. After $24 \mathrm{~h}$, cells were selected with $20 \mu \mathrm{g} / \mathrm{mL}$ blasticidin $\mathrm{S}$ for 14 days, and treated with $1 \mu \mathrm{g} / \mathrm{mL}$ doxycycline for 1 day to induce Cas9 expression. For preparing LFNG-KO Panc-1 cells, a lentiviral solution was used to infect the Cas9 stable Panc- 1 cells for $12 \mathrm{~h}$. After $12 \mathrm{~h}$, cells were treated with $1 \mu \mathrm{g} / \mathrm{mL}$ doxycycline for 1 day to induce Cas 9 expression. The transduction efficiency was confirmed by monitoring GFP expression. Both EOGTand LFNG-KO cells were cultured in DMEM/10\% FBS medium containing penicillin and streptomycin. A single cell was isolated by limiting dilution and seeding into 96-well plates for clonal selection and further analysis. 


\subsection{Evaluation of CRISPR/Cas9-mediated Genome Editing by T7 Endonuclease Assay and Sequencing Analysis}

Genomic DNA was extracted from wild-type Panc-1, clonally selected EOGT-KO Panc-1, and LFNG-KO Panc-1 cells. Briefly, all cells were cultured in 12-well plates. After harvesting, cells were centrifuged and washed with phosphate-buffered saline (PBS). Cells were heated at $95^{\circ} \mathrm{C}$ for $20 \mathrm{~min}$ after adding $18 \mu \mathrm{L}$ of $50 \mu \mathrm{M} \mathrm{NaOH}$ for each well of 12-well plates. Then, samples were vortex briefly, added with $3 \mu \mathrm{L}$ of $1 \mathrm{M}$ Tris- $\mathrm{HCl}, \mathrm{pH} 7.6$, and centrifuged at 15,000 rpm for $6 \mathrm{~min}$ after vortex to collect the supernatant. The target region was amplified with sgRNA-specific primers according to the manufacturer protocol. To detect double-stranded DNA mismatches and mutations, we used T7 endonuclease according to manufacture protocol. After confirming the knockout cells, single cells were isolated by limiting dilution. For EOGT-KO, of the 22 single-cell clones picked out, 10 clones showed negative expression of EOGT in Western blotting screening (Supplementary Figure S1B). We selected four clones for further analysis. To select LFNG-KO clones, the target regions of both wild-type and knockout cells were sequenced by Sanger sequencing. Four clones were selected for LFNG-KO Panc-1 cells after comparing with the wild-type sequence (Supplementary Figure S3).

\subsection{Immunostaining}

Panc-1 cells were fixed with $4 \%$ paraformaldehyde in PBS for $15 \mathrm{~min}$ and ice-cold methanol for $5 \mathrm{~min}$. Then cells were permeabilized with $0.5 \%$ Triton-X 100 in PBS for 15 min and blocked with 5\% BSA in PBS. Cells were incubated with Anti-AER61 (1:200) antibody diluted with $5 \%$ BSA/PBS overnight at $4{ }^{\circ} \mathrm{C}$. After washing with PBS, cells were incubated with DyLight 549-conjugated goat anti-rabbit IgG antibody (1:1000) diluted with $5 \%$ BSA/PBS for $1 \mathrm{~h}$ at room temperature. Then, cells were counter-stained with DAPI (Southern Biotech, Birmingham, AL, USA). All the slides were examined by TiEA1R Confocal Microscopy with NIS Elements (Nikon, Japan) [52].

\subsection{Western Blotting}

Cells were lysed in a lysis buffer containing Tris-buffered saline (TBS), pH 7.6, $1 \mathrm{mM}$ $\mathrm{CaCl}_{2}$, and $1 \%$ Nonidet P-40. Each sample was separated by 7.5\% SDS-PAGE for Western blotting and transferred onto a PVDF membrane (Merck, Japan). The membrane was blocked in 3\% BSA/PBST at room temperature for $1 \mathrm{~h}$, followed by incubation with antiAER61 antibody $(1: 10,000)$ diluted in 3\% BSA/PBST for $2 \mathrm{~h}$. After washing with PBST three times, the membrane was incubated with Anti Rabbit HRP conjugated secondary antibody $(1: 8000)$ in $3 \%$ BSA/PBST at room temperature for $1 \mathrm{~h}$. After washing with PBST, bands on the membrane were visualized with Immobilon Western chemiluminescent HRP substrate (Merck) and iBright FL1500 Imaging System (Thermo Fisher).

\subsection{Cell Proliferation Assay}

Cells were seeded 96-well plates at $7 \times 10^{3}$ cells per well in DMEM medium containing $10 \%$ FBS. The increasing confluence of the cells was measured as an indicator of cell proliferation using IncuCyte ZOOM Live-Cell Analysis System. Images were taken at $6 \mathrm{~h}$ intervals with a $4 \times$ objective from 3 separate wells, and mean \pm SD of confluence percentage was measured.

\subsection{Cell Migration Assay}

Cell migration (wound healing) assay was performed as reported previously [53]. Briefly, cells $\left(2 \times 10^{4}\right)$ were seeded into an uncoated 96-well plate image lock plate (Sartorius). After 6-8 h later, the wound was made using 96-pin WoundMaker (Sartorius). The images of the wound were taken every $2 \mathrm{~h}$ for $18 \mathrm{~h}$. Then, data were analyzed by calculating the relative wound density with IncuCyte ZOOM Live-Cell Analysis System. 


\subsection{Statistical Analysis and Database Analysis}

The significance of the data in functional assays was analyzed with one-way repeated measures ANOVA followed by Dunnett's Post-hoc test to show a significant difference between groups. Significance was evaluated based on the $p$-value. Gene expression and correlation were analyzed using the GEPIA2 [54] database for pancreatic adenocarcinoma (PAAD), in which the pre-processed RNA-seq datasets and a normalization method that calculates transcripts per million (TPM) were utilized. Survival analyses were analyzed with a TCGA dataset and EZR software (version 1.52), which is a graphical user interface for R software (version 4.0.2).

Supplementary Materials: Supplementary materials are available online. Supplementary Figure S1, characterization of EOGT-KO Panc-1 cells; Supplementary Figure S2, the inhibitory effect of DAPT on the proliferation of Panc-1 cells; Supplementary Figure S3, sequencing data of wild-type and LFNG-KO Panc-1 cells; Supplementary Table S1, primers and synthetic oligonucleotides used for Crispr/Cas9-mediated genome editing.

Author Contributions: R.B.: designed the overall project, generated all materials, performed experiments, data analysis, writing — original draft preparation; K.M.: data analysis; Y.T.: designed the overall project, funding acquisition; M.O.: provided reagents, funding acquisition; H.T.: provided reagents, funding acquisition; A.T.: designed the overall project, data analysis, writing—review, editing; T.O.: designed the overall project, data analysis, funding acquisition, writing—review, editing. All authors have read and agreed to the published version of the manuscript.

Funding: This work was supported by grants from the Japan Society for the Promotion of Science (JP19K16073 to M.O., JP19KK0195 and JP19H03176 to H.T., and JP19H03416 T.O.); the Foundation for Promotion of Cancer Research in Japan (to M.O.); the Hori Sciences and Arts Foundation (to M.O.); the Sasakawa Scientific Research Grant from Japan Science Society (to M.O.); the Takeda Science Foundation (to H.T. and M.O.), and the Mitsubishi Foundation (T.O.).

Institutional Review Board Statement: Ethical review and approval were waived for this study because the analysis of de-identified, publicly available data does not constitute human subjects research.

Informed Consent Statement: Patient consent was waived because the reason mentioned above.

Data Availability Statement: The data presented in this study are available in Supplementary Materials.

Acknowledgments: We would like to thank W. Saiki (Nagoya Univ) for providing critical reagents for the project. We also thank S. M. D. Alam and E.-T. Wan. We would like to thank E. Yorifuji at the Division for Medical Research Engineering, Nagoya University Graduate School of Medicine, for her technical support in operating the IncuCyte ZOOM system and TiEA1R Confocal Microscopy. We would also like to thank the members of the Okajima laboratory for their helpful discussions.

Conflicts of Interest: The authors declare no conflict of interest.

\section{References}

1. Bray, F.; Ferlay, J.; Soerjomataram, I.; Siegel, R.L.; Torre, L.A.; Jemal, A. Global cancer statistics 2018: GLOBOCAN estimates of incidence and mortality worldwide for 36 cancers in 185 countries. CA Cancer J. Clin. 2018, 68, 394-424. [CrossRef] [PubMed]

2. Siegel, R.L.; Miller, K.D.; Jemal, A. Cancer statistics, 2020. CA Cancer J. Clin. 2020, 70, 7-30. [CrossRef] [PubMed]

3. Vincent, A.; Herman, J.; Schulick, R.; Hruban, R.H.; Goggins, M. Pancreatic cancer. Lancet 2011, 378, 607-620. [CrossRef]

4. Kleeff, J.; Korc, M.; Apte, M.; La Vecchia, C.; Johnson, C.D.; Biankin, A.V.; E Neale, R.; Tempero, M.; Tuveson, D.A.; Hruban, R.H.; et al. Pancreatic cancer. Nat. Rev. Dis. Prim. 2016, 2, 16022. [CrossRef]

5. Bliss, L.A.; Witkowski, E.R.; Yang, C.J.; Tseng, J.F. Outcomes in operative management of pancreatic cancer. J. Surg. Oncol. 2014, 110, 592-598. [CrossRef]

6. Ntziachristos, P.; Lim, J.S.; Sage, J.; Aifantis, I. From Fly Wings to Targeted Cancer Therapies: A Centennial for Notch Signaling. Cancer Cell 2014, 25, 318-334. [CrossRef]

7. Tashima, Y.; Okajima, T. Congenital diseases caused by defective O-glycosylation of Notch receptors. Nagoya J. Med Sci. 2018, 80, 299-307.

8. Espinoza, I.; Miele, L. Notch inhibitors for cancer treatment. Pharmacol. Ther. 2013, 139, 95-110. [CrossRef] 
9. Miele, L.; Espinoza, I.; Pochampally, R.; Watabe, K.; Xing, F. Notch signaling: Targeting cancer stem cells and epithelial-tomesenchymal transition. OncoTargets Ther. 2013, 6, 1249-1259. [CrossRef] [PubMed]

10. Avila, J.L.; Kissil, J.L. Notch signaling in pancreatic cancer: Oncogene or tumor suppressor? Trends Mol. Med. 2013, 19, 320-327. [CrossRef]

11. Nakhai, H.; Siveke, J.T.; Klein, B.; Mendoza-Torres, L.; Mazur, P.K.; Algül, H.; Radtke, F.; Strobl, L.; Zimber-Strobl, U.; Schmidt, G. Conditional ablation of Notch signaling in pancreatic development. Development 2008, 135, 2757-2765. [CrossRef]

12. Hanlon, L.; Avila, J.L.; Demarest, R.M.; Troutman, S.; Allen, M.; Ratti, F.; Rustgi, A.K.; Stanger, B.Z.; Radtke, F.; Adsay, V.; et al. Notch1 Functions as a Tumor Suppressor in a Model of K-ras-Induced Pancreatic Ductal Adenocarcinoma. Cancer Res. 2010, 70, 4280-4286. [CrossRef] [PubMed]

13. Agrawal, N.; Frederick, M.J.; Pickering, C.R.; Bettegowda, C.; Chang, K.; Li, R.J.; Fakhry, C.; Xie, T.-X.; Zhang, J.; Wang, J.; et al. Exome Sequencing of Head and Neck Squamous Cell Carcinoma Reveals Inactivating Mutations in NOTCH1. Science 2011, 333, 1154-1157. [CrossRef]

14. Kopan, R.; Ilagan, M.X.G. The Canonical Notch Signaling Pathway: Unfolding the Activation Mechanism. Cell 2009, 137, $216-233$. [CrossRef]

15. Plentz, R.; Park, J.; Rhim, A.D.; Abravanel, D.; Hezel, A.F.; Sharma, S.V.; Gurumurthy, S.; Deshpande, V.; Kenific, C.; Settleman, J.; et al. Inhibition of $\gamma$-Secretase Activity Inhibits Tumor Progression in a Mouse Model of Pancreatic Ductal Adenocarcinoma. Gastroenterology 2009, 136, 1741-1749.e6. [CrossRef] [PubMed]

16. Miyamoto, Y.; Maitra, A.; Ghosh, B.; Zechner, U.; Argani, P.; A Iacobuzio-Donahue, C.; Sriuranpong, V.; Iso, T.; Meszoely, I.M.; Wolfe, M.S.; et al. Notch mediates TGF $\alpha$-induced changes in epithelial differentiation during pancreatic tumorigenesis. Cancer Cell 2003, 3, 565-576. [CrossRef]

17. Ye, J.; Wen, J.; Ning, Y.; Li, Y. Higher notch expression implies poor survival in pancreatic ductal adenocarcinoma: A systematic review and meta-analysis. Pancreatology 2018, 18, 954-961. [CrossRef]

18. Thomas, M.M.; Zhang, Y.; Mathew, E.; Kane, K.T.; Maillard, I.; Di Magliano, M.P. Epithelial Notch signaling is a limiting step for pancreatic carcinogenesis. BMC Cancer 2014, 14, 862. [CrossRef] [PubMed]

19. Song, H.; Wang, Y.; Lan, H.; Zhang, Y. Expression of Notch receptors and their ligands in pancreatic ductal adenocarcinoma. Exp. Ther. Med. 2018, 16, 53-60. [CrossRef] [PubMed]

20. Ma, J.; Xia, J.; Miele, L.; Sarkar, F.H.; Wang, Z. Notch Signaling Pathway in Pancreatic Cancer Progression. Pancreat. Disord. Ther. 2013, 3, 3. [CrossRef]

21. Matsuura, A.; Ito, M.; Sakaidani, Y.; Kondo, T.; Murakami, K.; Furukawa, K.; Nadano, D.; Matsuda, T.; Okajima, T. O-LinkedNAcetylglucosamine Is Present on the Extracellular Domain of Notch Receptors. J. Biol. Chem. 2008, 283, 35486-35495. [CrossRef]

22. Tashima, Y.; Stanley, P. Antibodies That Detect O-Linked $\beta-\mathrm{d}-\mathrm{N}-$ Acetylglucosamine on the Extracellular Domain of Cell Surface Glycoproteins. J. Biol. Chem. 2014, 289, 11132-11142. [CrossRef] [PubMed]

23. Cohen, I.; Silberstein, E.; Perez, Y.; Landau, D.; Elbedour, K.; Langer, Y.; Kadir, R.; Volodarsky, M.; Sivan, S.; Narkis, G.; et al. Autosomal recessive Adams-Oliver syndrome caused by homozygous mutation in EOGT, encoding an EGF domain-specific O-GlcNAc transferase. Eur. J. Hum. Genet. 2013, 22, 374-378. [CrossRef]

24. Ogawa, M.; Okajima, T. Structure and function of extracellular O-GlcNAc. Curr. Opin. Struct. Biol. 2019, 56, 72-77. [CrossRef]

25. Hassed, S.; Li, S.; Mulvihill, J.; Aston, C.; Palmer, S. Adams-Oliver syndrome review of the literature: Refining the diagnostic phenotype. Am. J. Med Genet. Part A 2017, 173, 790-800. [CrossRef]

26. Sawaguchi, S.; Varshney, S.; Ogawa, M.; Sakaidani, Y.; Yagi, H.; Takeshita, K.; Murohara, T.; Kato, K.; Sundaram, S.; Stanley, P.; et al. O-GlcNAc on NOTCH1 EGF repeats regulates ligand-induced Notch signaling and vascular development in mammals. eLife 2017, 6, 7280. [CrossRef] [PubMed]

27. Dunwoodie, S.L. Mutation of the fucose-specific $\beta 1,3 \mathrm{~N}$-acetylglucosaminyltransferase LFNG results in abnormal formation of the spine. Biochim. Biophys. Acta BBA Mol. Basis Dis. 2009, 1792, 100-111. [CrossRef] [PubMed]

28. Kakuda, S.; Lopilato, R.K.; Ito, A.; Haltiwanger, R.S. Canonical Notch ligands and Fringes have distinct effects on NOTCH1 and NOTCH2. J. Biol. Chem. 2020, 295, 14710-14722. [CrossRef]

29. Zhang, S.; Chung, W.-C.; Xu, K. Lunatic Fringe is a potent tumor suppressor in Kras-initiated pancreatic cancer. Oncogene 2016, 35, 2485-2495. [CrossRef] [PubMed]

30. Ogawa, M.; Senoo, Y.; Ikeda, K.; Takeuchi, H.; Okajima, T. Structural Divergence in O-GlcNAc Glycans Displayed on Epidermal Growth Factor-like Repeats of Mammalian Notch1. Molecules 2018, 23, 1745. [CrossRef]

31. Varshney, S.; Stanley, P. Multiple roles for O-glycans in Notch signalling. FEBS Lett. 2018, 592, 3819-3834. [CrossRef]

32. Kakuda, S.; Haltiwanger, R.S. Deciphering the Fringe-Mediated Notch Code: Identification of Activating and Inhibiting Sites Allowing Discrimination between Ligands. Dev. Cell 2017, 40, 193-201. [CrossRef] [PubMed]

33. Haltom, A.R.; Jafar-Nejad, H. The multiple roles of epidermal growth factor repeatO-glycans in animal development. Glycobiology 2015, 25, 1027-1042. [CrossRef] [PubMed]

34. Juiz, N.; Elkaoutari, A.; Bigonnet, M.; Gayet, O.; Roques, J.; Nicolle, R.; Iovanna, J.L.; Dusetti, N. Basal-like and classical cells coexist in pancreatic cancer revealed by single-cell analysis on biopsy-derived pancreatic cancer organoids from the classical subtype. FASEB J. 2020, 34, 12214-12228. [CrossRef] [PubMed]

35. Nandagopal, N.; Santat, L.A.; Lebon, L.; Sprinzak, D.; Bronner, M.E.; Elowitz, M.B. Dynamic Ligand Discrimination in the Notch Signaling Pathway. Cell 2018, 172, 869-880.e19. [CrossRef] 
36. Lopez-Fernandez, A.; Rodriguez-Baena, D.; Gomez-Vela, F.; Divina, F.; Garcia-Torres, M. A multi-GPU biclustering algorithm for binary datasets. J. Parallel Distrib. Comput. 2021, 147, 209-219. [CrossRef]

37. Orzechowski, P.; Sipper, M.; Huang, X.; Moore, J.H. EBIC: An evolutionary-based parallel biclustering algorithm for pattern discovery. Bioinformatics 2018, 34, 3719-3726. [CrossRef]

38. Xie, J.; Ma, A.; Zhang, Y.; Liu, B.; Cao, S.; Wang, C.; Xu, J.; Zhang, C.; Ma, Q. QUBIC2: A novel and robust biclustering algorithm for analyses and interpretation of large-scale RNA-Seq data. Bioinformatics 2020, 36, 1143-1149. [CrossRef]

39. Bhattacharya, A.; Cui, Y. A GPU-accelerated algorithm for biclustering analysis and detection of condition-dependent coexpression network modules. Sci. Rep. 2017, 7, 1-9. [CrossRef]

40. Du, X.; Cheng, Z.; Li, Y.; Zhou, Z.-G.; Yang, L.; Zhang, M.-M. Suppressive effects of gamma-secretase inhibitor DAPT on the proliferation of pancreatic cancer cells. Sichuan da xue xue bao. Yi xue ban J. Sichuan Univ. Med Sci. Ed. 2013, 44, 699-702.

41. Harbuzariu, A.; Rampoldi, A.; Daley-Brown, D.S.; Candelaria, P.; Harmon, T.L.; Lipsey, C.C.; Beech, D.J.; Quarshie, A.; Ilies, G.O.; Gonzalez, R.R. Leptin-Notch signaling axis is involved in pancreatic cancer progression. Oncotarget 2016, 8, 7740-7752. [CrossRef]

42. Hu, Y.; Su, H.; Ling, C.; Guoliang, Q.; Cheng, L.; Qin, R.; Qing, G.; Liu, H. The NOTCH Ligand JAGGED2 Promotes Pancreatic Cancer Metastasis Independent of NOTCH Signaling Activation. Mol. Cancer Ther. 2015, 14, 289-297. [CrossRef]

43. Chen, D.; Wang, H.; Bao, Y.; Xie, K. Notch signaling molecule is involved in the invasion of MiaPaCa2 cells induced by CoCl2 via regulating epithelial-mesenchymal transition. Mol. Med. Rep. 2018, 17, 4965-4972. [CrossRef] [PubMed]

44. Lieber, M.; Mazzetta, J.; Nelson-Rees, W.; Kaplan, M.; Todaro, G. Establishment of a continuous tumor-cell line (PANC-1) from a human carcinoma of the exocrine pancreas. Int. J. Cancer 1975, 15, 741-747. [CrossRef]

45. Watanabe, M. Metabolic Profiling Comparison of Human Pancreatic Ductal Epithelial Cells and Three Pancreatic Cancer Cell Lines using NMR Based Metabonomics. J. Mol. Biomarkers Diagn. 2012, 3. [CrossRef]

46. Single, A.; Beetham, H.; Telford, B.J.; Guilford, P.; Chen, A. A Comparison of Real-Time and Endpoint Cell Viability Assays for Improved Synthetic Lethal Drug Validation. J. Biomol. Screen. 2015, 20, 1286-1293. [CrossRef] [PubMed]

47. Naito, Y.; Hino, K.; Bono, H.; Ui-Tei, K. CRISPRdirect: Software for designing CRISPR/Cas guide RNA with reduced off-target sites. Bioinformatics 2015, 31, 1120-1123. [CrossRef]

48. Wang, T.; Wei, J.J.; Sabatini, D.M.; Lander, E.S. Genetic Screens in Human Cells Using the CRISPR-Cas9 System. Science 2014, 343, 80-84. [CrossRef] [PubMed]

49. Narimatsu, Y.; Joshi, H.J.; Yang, Z.; Gomes, C.; Chen, Y.-H.; Lorenzetti, F.C.; Furukawa, S.; Schjoldager, K.T.; Hansen, L.; Clausen, H.; et al. A validated gRNA library for CRISPR/Cas9 targeting of the human glycosyltransferase genome. Glycobiology 2018, 28, 295-305. [CrossRef]

50. Giuliano, C.J.; Lin, A.; Girish, V.; Sheltzer, J.M. Generating Single Cell-Derived Knockout Clones in Mammalian Cells with CRISPR/Cas9. Curr. Protoc. Mol. Biol. 2019, 128, e100. [CrossRef]

51. Abrahimi, P.; Chang, W.G.; Kluger, M.S.; Qyang, Y.; Tellides, G.; Saltzman, W.M.; Pober, J.S. Efficient Gene Disruption in Cultured Primary Human Endothelial Cells by CRISPR/Cas9. Circ. Res. 2015, 117, 121-128. [CrossRef] [PubMed]

52. Alam, S.M.D.; Tsukamoto, Y.; Ogawa, M.; Senoo, Y.; Ikeda, K.; Tashima, Y.; Takeuchi, H.; Okajima, T. N-Glycans on EGF domain-specific O-GlcNAc transferase (EOGT) facilitate EOGT maturation and peripheral endoplasmic reticulum localization. J. Biol. Chem. 2020, 295, 8560-8574. [CrossRef] [PubMed]

53. Libério, M.S.; Sadowski, M.C.; Soekmadji, C.; Davis, R.A.; Nelson, C.C. Differential Effects of Tissue Culture Coating Substrates on Prostate Cancer Cell Adherence, Morphology and Behavior. PLOS ONE 2014, 9, e112122. [CrossRef] [PubMed]

54. Tang, Z.; Kang, B.; Li, C.; Chen, T.; Zhang, Z. GEPIA2: An enhanced web server for large-scale expression profiling and interactive analysis. Nucleic Acids Res. 2019, 47, 556-560. [CrossRef] [PubMed] 